\title{
Le réseau des destins croisés
}

\section{Olivier Joseph}

\section{OpenEdition}

\section{Journals}

Édition électronique

URL : https://journals.openedition.org/rhcf/2063

DOI : 10.4000/rhcf.2063

Éditeur

Rails \& histoire

Édition imprimée

Date de publication : 2 mai 2002

Pagination : 245-269

ISBN : 00996-9403

ISSN : 0996-9403

\section{Référence électronique}

Olivier Joseph, «Le réseau des destins croisés », Revue d'histoire des chemins de fer [En ligne], 24-25 |

2002, mis en ligne le 15 avril 2015, consulté le 22 avril 2022. URL : http://journals.openedition.org/

rhcf/2063 ; DOl : https://doi.org/10.4000/rhcf.2063 


\section{Olivier JOSEPH \\ Le réseau des destins croisés}

La grille est désormais entièrement couverte par les tarots et les récits. Toutes les cartes ont été retournées sur la table. Même mon histoire y est comprise, bien que je ne sache plus dire laquelle c'est, tant est serré l'entrelacement de toutes à la fois. En fait, la tâche de déchiffrer les histoires une à une m'a fait jusqu'à présent négliger la particularité la plus saillante de notre mode de narration, à savoir que chaque récit court à la rencontre d'un autre, et tandis qu'un des convives progresse sur sa lancée, un autre parti de l'autre bout avance en sens opposé, puisque les histoires racontées de gauche à droite ou de bas en haut peuvent aussi être lues de droite à gauche ou de haut en bas, et vice versa, si on tient compte du fait que les mêmes cartes, en se présentant dans un ordre différent, changent de sens, et que le même tarot sert dans le même temps à des narrateurs qui partent des quatre points cardinaux.

Italo Calvino, Le Château des destins croisés.

Ce n'est ni dans un château, ni dans une auberge que se rencontrent les personnes qui, entre 1883 et 1893, décident la construction d'une ligne de chemins de fer secondaires entre Castres et la partie tarnaise du Haut-Languedoc, la Montagne, mais, conformément aux termes des lois de 1865 et 1880, dans l'enceinte du conseil général.

Nul sort n'est jeté qui réduit au silence et oblige ces élus à s'exprimer à l'aide de tarots, tels ceux subtilement mis en scène par Italo Calvino : les pages des délibérations du conseil général du Tarn, celles des journaux d'alors, les archives des Ponts et Chaussées bruissent d'un long débat, complexe parfois, houleux souvent.

Nombre de monographies historiques consacrées à ces lignes secondaires dont le souvenir est toujours vivace font état de longues discussions au sein des conseils généraux pour arriver aux décisions qui devaient être à l'origine de l'impressionnant développement des voies ferrées d'intérêt local en France au tournant du XIX ${ }^{\mathrm{e}}$ et du $\mathrm{XX}^{\mathrm{e}}$ siècles. Aucune - ou presque - ne s'y attarde.

L'histoire sociale et l'anthropologie des techniques, telle qu'elle est pratiquée par Thomas Hughes, Donald Mackenzie ou Bruno Latour ${ }^{1}$, par exemple, montrent la richesse qui se fait jour lorsque l'on suit pas à 
pas les acteurs engagés dans l'élaboration d'un projet technique, lorsque l'on prête attention à l'ensemble de leurs faits et gestes sans en éliminer apriori certains qui ne semblent pas directement concerner l'histoire des techniques. La fécondité de ces recherches commande d'adopter les mêmes méthodes d'analyse pour qui s'intéresse aux chemins de fers secondaires français.

Rendre aux débats du conseil général leur complexité technique et sociale, lester leurs acteurs de leurs itinéraires personnels, défaire, patiemment, la trame qui compose ces « destins croisés », cela permet-il de comprendre les ressorts et les enjeux de décisions qui marquèrent l'histoire des chemins de fer secondaires en France?

C'est à cette tâche que je me suis attelé pour le département du Tarn. L'étude qui suit ne donne qu'une faible idée des résultats de cette recherche : entre 1872 et 1897, les Monts de Lacaune ont fait l'enjeu de divers projets pour y établir des lignes de chemins de fer. À partir de 1883, le conseil général du Tarn, qui a déjà fort à faire à s'occuper des routes, du rachat des ponts, des épizooties ovines (une certaine tremblante du mouton), des écoles, de l'amélioration de l'agriculture, des vagabonds, des horaires des compagnies du Midi ou du P.O., des enfants trouvés..., va, par la volonté de certains de ses membres et les compétences attribuées par le législateur, s'intéresser à la question des chemins de fer secondaires.

Trois grandes périodes marquent les années 1883-1897, que je nomme : le temps des politiques, le temps des ingénieurs, le temps des concessionnaires. Les frontières entre ces trois temps ne sont étanches ni chronologiquement, ni humainement : elles se chevauchent parfois et les acteurs de chacune de ces trois périodes passent souvent de l'une à l'autre. En 1897, les trois temps et leurs acteurs se retrouvent ensemble, à la table des négociations, pour l'attribution de la concession! Mais cette délimitation reste pratique pour comprendre les moments clés de la construction de la décision.

$\mathrm{Si}$, dans mon intervention orale, $j$ ai pu passer en revue les principaux faits de chacun de ces trois temps, les remarques des professeurs Caron et Marnot m’ont encouragé à prendre à bras le corps ce que je n'avais fait qu'effleurer alors : l'analyse exhaustive de la décision du conseil général du Tarn.

Le nombre de faits et gestes des acteurs de cette décision que l'on peut reconstituer à l'aide des documents d'époque, la masse des archives disponibles permettent une étude d'une ampleur insoupçonnée, dont cet article ne peut rendre compte par manque de place. C'est donc uniquement au temps des politiques ${ }^{2}$ que je consacre ces quelques pages. 


\section{"Le petit rien qui nous divise"}

Lorsque la question des chemins de fer secondaires se pose sérieusement aux élus du conseil général du Tarn, c'est, lors de la session d'avril 1883, à un double titre : il s'agit, pour certains, de relier Graulhet à une ligne déjà existante ; pour d'autres, de créer un véritable réseau départemental secondaire qui servirait les cantons délaissés par les lignes existantes (dont celui de Graulhet).

L'injustice faite à Graulhet devait être réparée : alors que la ligne projetée pour relier Castres à Montauban pouvait emprunter la vallée du Dadou, le choix s'était fixé sur celle de l'Agout, desservant Lavaur et négligeant Graulhet, l'une des villes les plus industrieuses du Tarn grâce à son florissant commerce des peaux. Les vœux réitérés dans l'enceinte du conseil général, les études, promises mais toujours retardées, semblant ne pas suffire, l'hiver 1882-1883 est mis à profit pour une série de grandes manœuvres : les votes de subventions par les conseils municipaux de Graulhet et de six communes avoisinantes n'ont d'autre objectif que d'inciter le conseil général à prendre une décision ferme. Ils dessinent, dans le même temps, l'itinéraire que devra suivre cette voie ferrée : partant de Graulhet, la ligne se dirigera vers l'Ouest pour se rattacher au réseau du Midi aux alentours de Lavaur.

À presque 74 ans, Paul Abrial père, le conseiller général de Graulhet, met en avant l'urgence d'une décision et son caractère d'intérêt général, soutenu par le préfet, Léon Bourgeois.

Il y a urgence, certes, et tous s'accordent à le dire : Laportalière, conseiller général de Réalmont, reconnait qu' "il est incontestable que la ville de Graulhet est une ville manufacturière très importante »; le baron René Reille, qui défend les intérêts du canton de Saint-Amans-Soult, à l'est du département, en rajoute sur «la nécessité qu'il y a de mettre cette ville en relation avec les grands centres d'industrie »; Dubernard, venu des contreforts des Monts de Lacaune, abonde. Barbey, maire et conseiller général de Mazamet, récent sénateur du Tarn, se fait fort de rattacher le sort de Graulhet à l'ensemble des cantons qui jouxtent le sien :

«Il n'y a pas dans le Tarn une exploitation de laine, je pourrais presque dire une manufacture de draps, qui ne soit plus ou moins en rapport avec cette ville. »

Car c'est bien tout le sud du Tarn qui est lié, par le délainage, la mégisserie, les filatures, à la puissance économique du tandem MazametGraulhet, que représentent ici Barbey et Abrial, et dont le chiffre d'affaires dépasse de dix fois celui des mines de Carmaux! Quant au préfet Léon Bourgeois, acquis à l'urgence et à l'importance de la liaison ferroviaire, il pousse le conseil à prendre une décision hardie : 
« [...] je demande au conseil, qui connait l'importance économique de Graulhet, de son trafic, le chiffre de ses affaires, qui s'élève à 15 millions, ses relations avec les grands centres industriels du Midi de la France, de vouloir bien considérer qu'il ne s'agit pas d'un de ces petits intérêts locaux qu'on satisfait par la voie étroite, mais d'un intérêt général qu'on satisfait par la voie large. »

L’urgence et l'absolue nécessité d'accorder à Graulhet ce que l'intérêt général commande ne sauraient désarmer ceux des membres de l'assemblée départementale qui ont d'autres visées.

Le préfet le sait bien d'ailleurs, qui souligne ce «petit rien qui nous divise » mais hésite pourtant sur sa portée réelle, oscillant entre un « petit rien » qui peut être « divergences profondes » ou « accord presque complet $» \ldots$.

Ce "petit rien », Caussé, rapporteur de la Commission départementale, l'avait signalé :

«Faut-il faire étudier une voie large [...] ? Ne suffirait-il pas d'un chemin à voie étroite? »

Dévider la pelote constituée autour de ce "petit rien » c'est entrer au cœur du conflit qui va agiter le conseil général pendant quelques années...

Barbey et Abrial, épaulés par le préfet, ne démordent pas de la voie normale qui, seule, permettra le transport des marchandises vers Marseille, Bordeaux ou Paris sans rupture de charge et, donc sans surcoûts liés aux transbordements lors de l'arrivée des wagons sur le réseau de la Compagnie du Midi. Ajoutant les subventions acquises aux promesses et demandes (100 000 francs pour Graulhet, 33000 pour les autres communes, 500000 demandés au conseil général), ils sont convaincus qu'une compagnie privée pourra se charger de construire et d'exploiter les 14 kilomètres qui raccorderont Graulhet à la ligne Castres-SaintSulpice pour un coût estimé à 1753600 francs.

Mais certains conseillers généraux ont d'autres intérêts à défendre :

«Les soussignés prient le Conseil Général d'émettre le vœu suivant : "Le Conseil général, considérant que l'absence de toute voie ferrée dans la partie montagneuse de l'arrondissement de Castres et l'éloignement auquel se trouvent les gares existantes placent cette vaste région industrielle et agricole dans un état d'infériorité qui arrête son développement et préjudicie dans une large mesure aux intérêts publics et privés, renouvelle les vœux par lui antérieurement émis et prie instamment le gouvernement : $1^{\circ}$ de faire procéder à la construction de la ligne allant d'Estréchoux au bassin houiller de Castanet-le-Haut ; $2^{\circ}$ de comprendre dans les chemins à créer le prolongement de cette voie ferrée jusqu’à Castres."

Signé : Cambon, Vergnes, Dubernard. » 
Ce n'est pas la première fois que les représentants des cantons de Lacaune, Murat et Brassac voient dans le prolongement de la ligne de Béziers à Graissessac vers Estréchoux, Castanet-le-Haut et Castres l'occasion d'un désenclavement ferroviaire de la Montagne ; ce n'est pas la première fois non plus qu'ils en font la demande au conseil général, où personne ne semble mettre en doute la nécessité de leur vœu :

«Barbey - Je pense [...] que la ligne entre Castres et Castanet-leHaut [...] rendra des services considérables à la partie montagneuse de notre département privée jusqu’à ce jour de voies ferrées. »

Barbey presse cependant le conseil de ne s'intéresser pour l'heure qu'à la seule desserte de Graulhet.

Voter immédiatement 500000 francs pour la réalisation dans les plus brefs délais du chemin desservant Graulhet, n'est-ce pas prendre le risque de lier pour longtemps les finances du département à une seule ligne?

« De Laportalière - Dans ce vote de 500000 francs, les lignes à petite voie ne seraient pas comprises, ce me semble, et cela est injuste ; il est incontestable que la ville de Graulhet est une ville manufacturière très importante et qu'elle n'est pas desservie par un chemin de fer; que, par conséquent, les intérêts de cette ville amènent le Conseil général à s'intéresser à elle; mais il n'en n'est pas moins vrai qu'il y a aussi beaucoup d'intéressés qui se trouvent dans une foule de cantons ; il ne faut pas localiser l'intérêt général, il faut que tout le monde puisse en bénéficier. »

L'intervention du conseiller général de Réalmont n’est pas mince. Située à quatre kilomètres de la gare de Laboutarié, sur la ligne Midi qui relie Albi à Castres, la petite cité de Réalmont, siège d'un important marché, proche des mines de Peyrebrune et porte de pénétration dans la Montagne, se pose en concurrente - en rivale ? - des communes situées à l'ouest de Graulhet, ville dont elle n'est distante que de dix-sept kilomètres. Pourquoi, estiment certains, Laportalière en tête, relier Graulhet à la ligne Castres-Saint-Sulpice alors qu'il serait rationnel de se diriger vers Laboutarié pour aboutir plus directement à l'arrondissement de Castres ? Pourquoi hésiter, alors que, de Graulhet à Laboutarié, treize kilomètres s'offrent quasiment en ligne droite et sans dénivellation, sans aucun ouvrage d'art, en accotement continu d'une route départementale, ce qui n'est pas le cas en direction de Fiac ou de Lavaur ? Et que coûtent quatre kilomètres supplémentaires à établir dans les mêmes conditions, avec, à la clé, le trafic que générera Réalmont? 
La question revient, invariable : suivre Barbey, Abrial père et le préfet Bourgeois dans leur demande immédiate, n'est-ce pas mettre en péril tous les autres projets ? N'est-ce pas renoncer à «l'idée initiale, l'idée mère » (Bourgeois) : un réseau départemental ? Faut-il préférer une liaison à l'Ouest, à travers le territoire du seul canton de Graulhet ? Ou, partant vers l'Ouest, mettre dans la balance un second canton, celui de Réalmont et sa position charnière dans la géographie et l'économie du département?

Barbey et Abrial père restent fermes sur leurs positions... Le préfet tente de concilier les avis tout en restant décidé à satisfaire les demandes de Graulhet.

À l'heure des décisions, un compromis peut-il se dessiner? Léon Bourgeois prend l'initiative d'un vote dont il rédige les différentes propositions. Si l'assemblée départementale accepte que soit étudié, dans son ensemble, un réseau ; si elle accepte aussi que la ligne desservant Graulhet soit étudiée en priorité, elle refuse clairement qu'elle le soit à voie large.

Présentés sur un carte, les votes des 24 conseillers généraux qui s'expriment (sur 35) semblent clairs pour ceux qui s'opposent à la voie large : ils sont majoritairement situés sur le tracé du réseau départemental (fig. 1). Les votes des promoteurs de la voie large recoupent, pour partie, les intérêts économiques des cantons de Graulhet et de Mazamet. Restent des votes qui semblent ne pas correspondre aux enjeux géographiques perçus à la lecture des délibérations du conseil général.

Une lecture synoptique de cette première carte et de celle indiquant l'appartenance politique des conseillers généraux en avril 1883 (fig. 2) donne à ce vote un autre sens : opte pour la voie large et la priorité à Graulhet, une large majorité de républicains (seuls Abrial père et fils se joignent à eux : logique géographique et discipline familiale semblent expliquer ces deux votes) ; optent pour le réseau départemental, des conservateurs (seuls les républicains Cambon à Lacaune et Falguière à Cadalen votent avec eux : ici la logique géographique semble primer!).

Le vote d'avril 1883 marque la victoire du projet porté par les conservateurs, majoritaires au conseil général, sur celui des républicains, minoritaires. Le soutien appuyé du préfet à ces derniers ne permet pas de renverser la situation.

Les élections cantonales d'août 1883 vont modifier profondément le paysage politique : «La délivrance de notre département est complète à présent. Vive la République !» claironne Le Réveil du Tarn 
du 19 août 1883, le journal d'Édouard Barbey, qui célèbre la majorité républicaine acquise d'un siège.

L'ouverture de la session du conseil général, le 21 août, permet à Barbey d'accéder à la présidence de l'assemblée. «Président de hasard, élu par une majorité de hasard » commente, amer, Le Conservateur, journal de René Reille.

Les républicains constatent et préviennent : «Les monarchistes du conseil général paraissent fort abattus [...] M. Reille est moins remuant [...] nos amis aujourd'hui les plus forts triomphent modestement et n'ont pas, il s'en faut de beaucoup, la morgue et l'arrogance qu'avaient les maitres d’hier. » (Réveil, 26 août 1883.)

La bataille qui va se dérouler dans l'enceinte du conseil général les 21 et 24 août montre cependant que les conservateurs ne sont pas «abattus ».

C'est un nouveau venu sur la scène du conseil général qui, à peine ouverte la session, se livre à une bataille éclair pour la ligne de Graulhet à voie large : Gabriel Compayré, député et conseiller général de Lavaur, fait adopter le principe de la création d'une Commission spéciale des chemins de fer et la désignation de ses sept membres par un scrutin secret. Il est immédiatement procédé au vote et, sans surprise, six républicains sont élus pour un seul conservateur.

Mais cette victoire a toutefois valeur d'avertissement : aucun des six républicains ne fait le plein des voix de son camp. Tous sont élus par 16 voix sur les 18 que compte la majorité !

Le seul conservateur élu l'est par 30 suffrages ! Ludovic de Naurois semble être porté par les 17 voix de son camp auxquelles se joindraient 13 voix républicaines! Nouveau venu, lui aussi, au conseil général, le maire de Lacaune est soutenu par la majorité des élus républicains alors qu'il est probablement parmi les plus agités des monarchistes, les plus farouches des conservateurs, et que le préfet Léon Bourgeois guette ses moindres gestes : il l'a révoqué de ses fonctions de maire en mars, et le révoquera, dans quelques jours, de ses fonctions de conseiller général. Il n'en reste pas moins que son investissement pour l'amélioration de la race ovine dans le Haut-Languedoc et le Rouergue voisin, pour la création des thermes de Lacaune, son acharnement à faire circuler sur les routes de la Montagne des trains routiers tractés par des locomotives Aveling \& Porter, son inlassable plaidoyer pour la création d'une ligne de chemin de fer de Castres à Lacaune le désignent comme le plus éminent conseiller général pour la question des chemins de fer secondaires. 


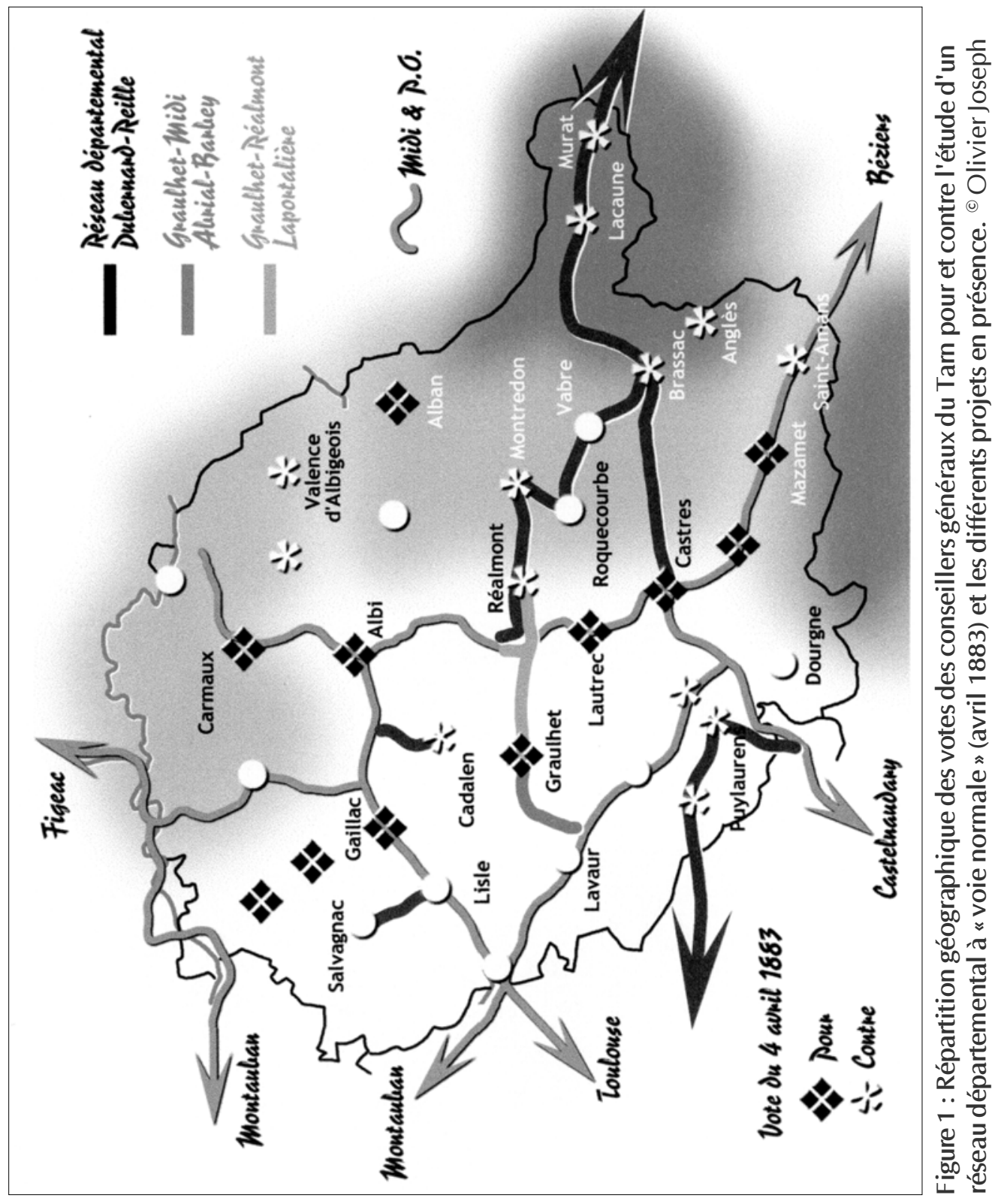




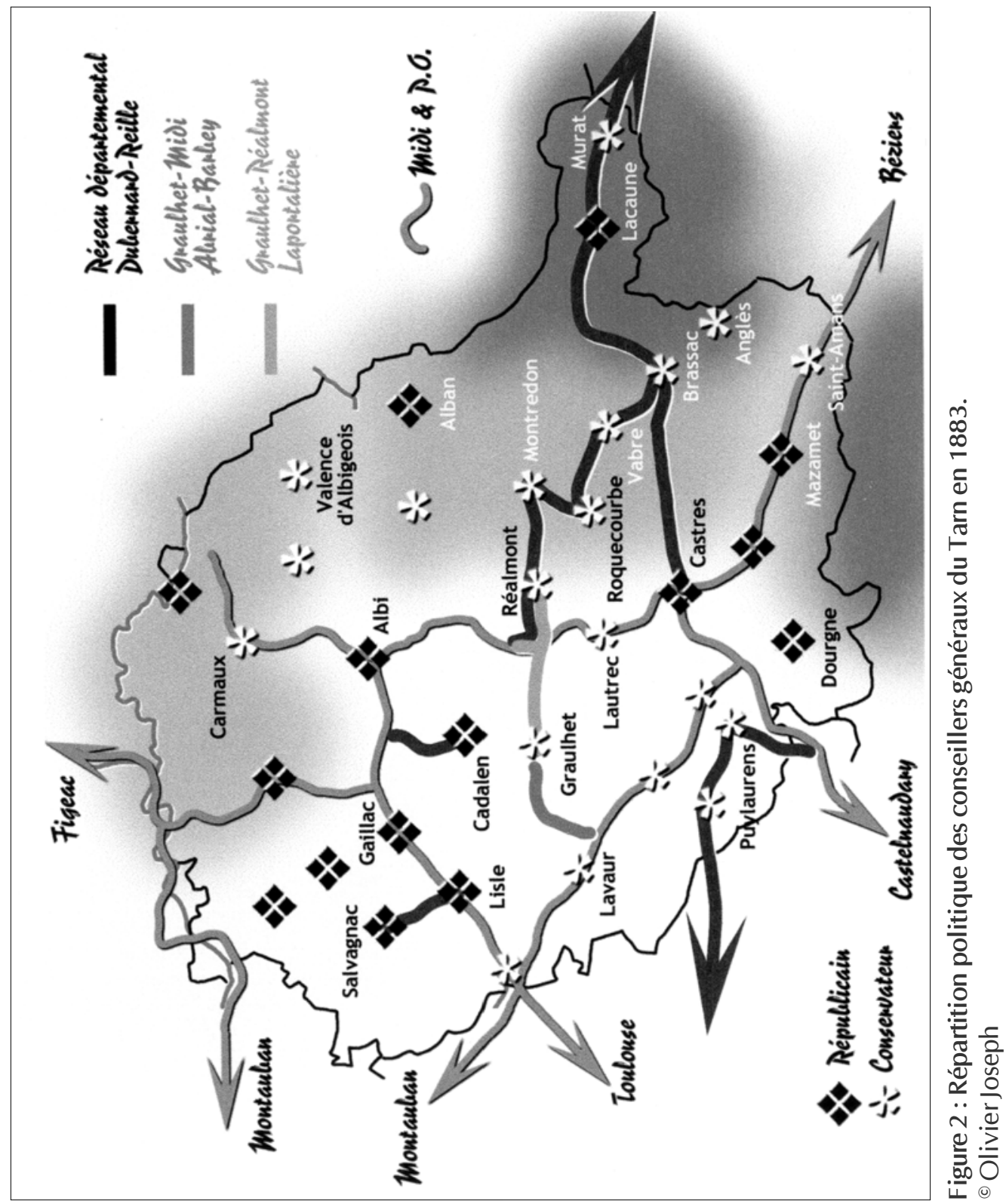


À six contre un, cependant, les républicains ont les coudées franches. Sans s'embarrasser du vote d'avril, Compayré propose, le 24 août, de prendre immédiatement la décision de construire la ligne de Graulhet, de la construire à voie large et de voter une subvention départementale de deux millions de francs.

Face à ce qui n'est autre qu'une tentative des républicains de passer en force, Reille et Dubernard mènent, pendant de longues heures, une bataille systématique contre le projet présenté par la Commission spéciale des chemins de fer : chaque point du rapport présenté par Compayré et soutenu par le préfet Léon Bourgeois est décortiqué, passé au crible de la critique, alternativement, par les deux élus conservateurs. Leur objectif reste le même qu'en avril : éviter que soit décidée la seule construction de la ligne de Graulhet, remettre au premier plan le réseau départemental, prendre des décisions pour l'ensemble du réseau, procéder aux études et déterminer les coûts du réseau, accorder à la ligne de Graulhet, comme position de compromis, la priorité de construction, mais non de concession.

À l'heure du vote qui doit clore la session d'août, la première manche est remportée par les conservateurs : c'est sur la proposition formulée par Dubernard, et non sur le rapport de Compayré, que se prononce l'assemblée. L'élu de Brassac demande le renvoi de la question des chemins de fer secondaires à la Commission départementale, l'étude détaillée du réseau par les services des Ponts et Chaussées. Son objectif est de provoquer les candidatures de compagnies privées qui se porteront concessionnaires de l'ensemble du réseau, avec, éventuellement, priorité de construction pour la ligne de Graulhet :

"Vous allez prendre, Messieurs, une décision très grave, car, si le réseau n'est pas concédé en bloc, la construction des lignes qui n'ont pas été étudiées encore n'aura jamais lieu. »

Trente et un conseillers généraux, sur 35 , prennent part au vote : la proposition Dubernard est adoptée à une majorité de 17 voix - ce qui équivaut au nombre de conseillers de son camp - contre 14. Mais quatre conseillers généraux républicains semblent avoir cautionné les propositions de la droite en s'abstenant ! voise :

L'éditorialiste du journal conservateur d'Albi, Le Nouvelliste, pa-

«La session d'août a été close avant-hier par deux séances publiques qui méritent [...] d'être contées. Mentionnons d'abord le rapport sur les chemins de fer qui donne pleinement satisfaction de la droite.[...] M. Compayré fait la grimace. Ajoutons d'ailleurs que le chemin de fer à voie large a été rejeté. On peut dès lors se 
faire une idée du désastre qui atteint le député de Lavaur. Ni la construction immédiate ni la voie large qu'il demandait n’ont été adoptés. » (2 septembre 1883)

Et poursuit :

«Bien qu'ils fussent en minorité, nos amis ont rendu d'éminents services à la cause du département, et ce qui le prouve, c'est que malgré les suspicions dont ils sont entourés, ils ont pu rallier à eux un petit groupe de Républicains. »

Sous la plume du journaliste conservateur, la description de l'affrontement au sein du conseil général rejoint ce que nous avions discerné : une opposition forte entre républicains et conservateurs, emmenés par Barbey, Compayré et le préfet Bourgeois pour les premiers, par Reille et Dubernard pour les seconds.

«Le petit rien » qui divise l'assemblée départementale est-il politique, technique ou économique ? un peu des trois à la fois ? ou franchement politique ? Fions-nous à Italo Calvino, et tentons de retrouver comment les destins des acteurs de cette controverse se croisent et se décroisent à propos des chemins de fer, comme à propos de tout autre chose : «les suspicions » dont sont entourés les conservateurs vont soudain prendre tout leur relief...

Lorsque Compayré prend la parole, il n'est pas un inconnu pour Reille ni pour Dubernard ; il était même, à l'occasion des élections qui viennent de se dérouler, l'homme à abattre :

«Là plus que partout ailleurs, la lutte aura une signification plus catholique que politique, plus religieuse que cantonale. Ce sera la lutte des croyances chrétiennes contre la morale indépendante et sans Dieu, du catholicisme contre les manuels de la libre pensée. Catholiques du canton de Lavaur, que pas un de vous ne manque au rendez-vous du scrutin. » (Conservateur, 2 août 1883.)

"Catholicisme », « libre pensée », « manuels scolaires »... Nous voici loin de la question des voies ferrées d'intérêt local. Nous sommes pourtant au cœur de ce qui agite les esprits.

Agrégé de philosophie, professeur à l'université de Toulouse puis à l'École normale supérieure de Fontenay-aux-Roses, élu député de Lavaur en 1880, Gabriel Compayré rédige, la même année, un manuel scolaire destiné à prendre place dans la vaste réforme scolaire engagée par Jules Ferry : les Éléments d'instruction morale et civique. Après la loi du 28 mars 1882, portant sur l'enseignement gratuit, obligatoire et laïque, le manuel Compayré est adopté par 50 départements français; il est alors tiré à 100000 exemplaires. La réputation de son auteur en fait le 
manuel le plus utilisé dans le Tarn, ce qui ne va pas sans provoquer le mécontentement d'une large partie du clergé tarnais, contre l'avis de l'archevêque d'Albi. Prélat modéré, plutôt enclin à composer avec la République qu'à chercher à l'abattre, Mgr Ramadié espère encore pouvoir trouver un compromis avec le gouvernement pour aménager la laïcité scolaire, en garantissant une stricte neutralité.

Mais, sur le terrain, des prêtres usent de pressions sur les parents d'élèves qui ont entre leurs mains le manuel Compayré, allant jusqu'à refuser les sacrements aux familles réfractaires. L'agitation gagne rapidement les communes tarnaises où certaines écoles de la République se retrouvent sans élèves...

Les efforts conciliateurs de Mgr Ramadié sont pris de court par une décision de Rome : la mise à l'Index, le 15 décembre 1882, de quatre manuels scolaires dont celui de Gabriel Compayré. Cédant aux pressions de son clergé et des notables catholiques du département, l'archevêque commente, dans une lettre pastorale datée du 24 février 1883, le texte de la condamnation de l'Index. Il légitime ainsi la bataille scolaire qui fait rage. Le geste de Mgr Ramadié donne une ampleur nationale à ce qui se déroule sur la scène politique, scolaire et religieuse tarnaise : d'autres évêques, d'autres départements entrent à leur tour dans le conflit.

La réaction du gouvernement ne se fait pas attendre. Pressé par le sénateur Bernard Lavergne, républicain combatif, le préfet Léon Bourgeois, arrivé à Albi le 8 novembre 1882, suspend les traitements de 50 prêtres (200 furent sanctionnés dans toute la France : le Tarn en représente donc le quart!), en déplace certains, révoque les maires qui font pressions sur les instituteurs, au premier rang desquels Ludovic de Naurois.

Aussi, lorsqu'en août 1883 Gabriel Compayré tente de passer en force pour imposer la ligne de Graulhet contre le réseau départemental, il le fait, croit-il, en vainqueur de la guerre scolaire et religieuse qui se déroule depuis quelques mois et dont son manuel est la principale cible. Les électeurs ne viennent-ils pas d'apporter leur soutien à la cause des républicains en faisant basculer le conseil général dans leur camp ?

C'est oublier que, malgré les sanctions prises par le préfet, les cléricaux tiennent bon. Ils vont montrer, lors de la session du conseil général, que leur pugnacité n'est pas entamée par la défaite électorale.

En compagnie de René Reille, c'est Dubernard, le conseiller général de Brassac, qui joue un rôle de premier plan pour la promotion et la défense inlassable du réseau départemental et, in fine, pour celle de la 
ligne de la Montagne. On retrouve aussi Dubernard dans toutes les discussions sur les routes et les chemins dont il semble être un spécialiste compétent à l'avis respecté.

Si Dubernard n'a laissé aucune trace dans la mémoire historique du Tarn, la perception de son action politique, par ses amis ou ses ennemis, livre quelques indices importants.

Le sous-préfet de Castres, dans un rapport de 1882, classe les Dubernard, avec les Reille, parmi les grandes familles bourgeoises « auxquelles les paysans sont restés attachés, un peu par une affection ancienne, et beaucoup par habitude ou par crainte, car on a toujours vu ces genslà au pouvoir et on croit qu'encore ils disposent d'une manière ou d'une autre d'une grande puissance ».

À l'occasion des cantonales de 1883, Le Conservateur se réjouit : « [Dubernard] n’a pas de concurrent et ne pourrait pas en avoir. Comme toujours, il ne sera pas élu mais acclamé. » (2 août 1883.)

Les républicains du Réveil du Tarn ne sont pas en reste : à sa mort, en 1896, Dubernard est qualifié de «lieutenant chéri» du baron Reille (23 août 1896). Lors des élections cantonales de 1889 les attaques des républicains sont plus précises encore :

«Savez-vous, électeurs, pourquoi M. Reille n'a rien fait pour vous au conseil général ? [...] parce qu'il préférait faire accorder à M. Dubernard les sommes qu'il aurait pu obtenir pour le canton de Saint-Amans. Avec ces sommes, M. Dubernard faisait construire des chemins dans le canton de Brassac et obtenait ainsi des voix à M. Reille pour la députation. 》 (Petit Républicain de SaintAmans, 28 août 1889.)

Liés par leur statut de grands bourgeois, Dubernard et Reille le sont aussi, dans l'esprit des républicains, par une alliance électorale qui s'exprime à travers la construction de chemins dans le canton du premier !

Chemins de terre, chemins de fer: dans un cas comme dans l'autre, dans la réalité comme dans l'esprit des républicains, Dubernard et Reille sont étroitement associés.

Installé à Saint-Amans-Soult, René Reille est, dans le Tarn, le plus éminent représentant de la noblesse d'empire, du bonapartisme, du catholicisme militant et de la grande bourgeoisie d'affaires : il est alors administrateur du P.O. et de la société des Mines de Carmaux. Élu au siège de député de la circonscription de Mazamet, il présente cette particularité de bénéficier du vote des ouvriers de l'industrie mazamétaine dont les dirigeants, protestants, sont parmi les plus importants 
représentants républicains du département : tel est le cas d’Édouard Barbey, sénateur, maire de Mazamet et président du conseil général depuis août 1883.

« Roi de la Montagne », « Homme de toutes les réactions », René Reille est, aussi, un des principaux animateurs de la querelle autour du manuel Compayré.

Aussi, lorsqu'en août 1883 Dubernard et Reille discutent pied à pied le rapport Compayré sur les voies ferrées d'intérêt local, ils le font « entourés » des « suspicions » des républicains et du préfet qui n’oublient pas qu'ils ont affaire à des empêcheurs de laïciser en rond !

L'intelligence politique de Dubernard et de Reille, leur parfaite maitrise du monde des chemins de fer (pour Reille), de la question des déplacements et des routes dans le Tarn (pour Dubernard) vont leur permettre de défaire le programme de Compayré en ramenant le conseil général aux précédentes décisions : des études approfondies pour l'ensemble des lignes demandées, la concession en bloc du réseau départemental, la priorité de construction, à voie large éventuellement mais non nécessairement, pour la ligne de Graulhet.

Compayré, fort de sa victoire électorale, exige et impose un règlement politique de la question des chemins de fer d'intérêt local. Il va ainsi au devant d'une défaite politique infligée par les vaincus du scrutin cantonal. Dubernard et Reille ne s'y trompent pas en ramenant les discussions sur le terrain de l'intérêt d'un maximum de cantons : ils y gagnent le soutien implicite de quatre conseillers généraux républicains et la cohésion sans faille du camp conservateur. Ce faisant, ils remportent, dans les circonstances précises que nous venons de développer, une victoire politique dont Le Nouvelliste souligne la portée !

Gabriel Compayré tentera une dernière fois de jouer un rôle clé dans la question ferroviaire, lors des sessions de 1884, avant de se taire et de céder définitivement la place à Édouard Barbey dans ce dossier.

À Rome, comme à Paris, cependant, on insiste sur la nécessaire «pacification religieuse » à l'heure où la France entreprend un vaste programme de laïcisation de ses institutions. Léon XIII, s'il a laissé agir la Congrégation de l'Index, n'a jamais condamné la République française. Entre juin et novembre 1883, Jules Ferry et Léon XIII vont trouver un compromis pour débloquer la situation. La conséquence immédiate, pour le Tarn, en est le rétablissement du traitement de la quasi-totalité des prêtres sanctionnés, la mutation de Léon Bourgeois dont l'activisme républicain avait fini par gêner le gouvernement français plus qu'il ne l'aidait. 
Les républicains sont déçus. Les cléricaux, eux, se laissent aller à quelques vers en guise de communiqué de victoire (Conservateur, 4 novembre 1883) :

«M. Bourgeois nous a quitté au moment de la chute des feuilles, quand l'hirondelle s'envole vers des climats plus doux.

L'oiseau que le printemps ramène

Par les froids d'octobre transi

Va bientôt quitter notre plaine...

Notre préfet nous quitte aussi.

$[\ldots]$

Salut à toi belle hirondelle !

Fuis loin de nos sombres frimas

Mais au printemps revient fidèle ! ...

Adieu Bourgeois, ne reviens pas...»

Malgré de nouvelles études, malgré de nouvelles tentatives républicaines de passer en force, malgré l'érosion des positions conservatrices, les positions des uns et des autres vont rester les mêmes durant les session du conseil général de 1884 et de 1885. La voie large et la priorité de concession pour Graulhet représentent toujours un point de blocage pour lequel le conseil général ne parvient pas à trouver de solution.

Édouard Barbey reconnait, avec ses « amis », que « trois fois nous sommes revenus à la charge, trois fois nous avons été battus ».

Au mois d'août 1885, cependant, c'est l'ingénieur en chef Hyppolite Pot qui va trouver le terrain du compromis : la voie de un mètre comme « réalisant l'ensemble des desiderata du Conseil ». Les conservateurs n'y sont pas totalement favorables mais, malgré une dernière opposition de Reille, Dubernard et Vergnes, le conseil général adopte le principe du réseau départemental et le vote immédiat des fonds pour la construction de la ligne de Graulhet. Barbey est satisfait et souligne que «cette modeste ligne de Graulhet à Laboutarié pourra devenir effectivement le point de départ et comme l'aurore du réseau départemental ».

Las... Le 11 juillet 1886, le conseil municipal de Graulhet annule sa subvention de 150000 francs car il refuse toute idée d'une desserte à voie étroite.

Le projet est enterré ! 


\section{«Cette sorte d'aurore de 1893 "}

SÉNAT

Paris, le 14 septembre 1893

Monsieur l'Ingénieur en Chef,

[...] Cette assemblée a manifesté en effet pour la première fois de la façon la plus nette son désir de mener à bonne fin l'étude des chemins de fer à voie étroite destinés à desservir les cantons privés de voies ferrées, sous la seule réserve que les projets seraient dressés dans des conditions d'économie aussi rigoureuses que possibles. On ne veut ni du grand ni du beau ni même du confortable ; on veut du bon marché, et c'est en effet le bon marché seul qui peut permettre la construction sinon de toutes les lignes au moins des deux ou trois principales.

Parmi elles se trouve, de beaucoup au premier rang, celle de Castres à Murat. Le conseil général en décidant qu'elle serait étudiée la première a bien montré qu'il attachait à cette voie de communication intéressant sept cantons privés de chemin de fer, c'est à dire le cinquième du territoire de notre département, une importance exceptionnelle, et les quatre mille francs qu'il a votés séance tenante représentent justement les frais d'étude qui la concernent.

J'estime donc que pour répondre aux vues de l'assemblée départementale et pour bien assurer à votre service la direction complète de ce que j'appellerai « le réseau Decauville du Tarn », il importe de se mettre immédiatement à l'œuvre et de profiter des deux mois de saison passable que nous allons traverser pour organiser les études dans la montagne et préparer les avant-projets.

Vous remarquerez en outre que le conseil général a spécifié que la préparation des projets et les étude statistiques propres à l'éclairer sur les revenus probables de l'exploitation marcheraient de front. C'est donc encore un point sur lequel j'appelle d'autant plus votre attention que ceux de nos collègues qui ne croient pas à la possibilité d'établir tout ou partie du réseau demandaient que les études statistiques fussent faites préalablement aux études techniques. Le conseil général a refusé d'adopter cette manière de voir indiquant ainsi une fois de plus sa ferme résolution d'aboutir.

J'espère, Monsieur l'Ingénieur en Chef, que vous voudrez bien partager ma manière de voir sur cette importante question. S'il en est ainsi, je m'empresserai de la faire connaître à $\mathrm{M}$. le Préfet qui a seul la charge et la responsabilité de l'exécution des délibérations du conseil général.

Recevez, je vous prie, Monsieur l'Ingénieur en chef, la nouvelle assurance de mes sentiments les plus distingués.

\section{Édouard Barbey}


La lettre expédiée par Édouard Barbey à l'ingénieur en chef Georges Callon marque, dix ans après les affrontements de 1883, un retournement spectaculaire dans l'élaboration du programme de chemins de fer secondaires du Tarn.

En 1892, réunis en « comité secret », les élus ont adopté le principe d'un réseau départemental. En quelques heures, ils ont donné satisfaction à Grauhlet, en accordant, sans opposition manifeste, la concession d'une ligne de Graulhet à Laboutarié, non en voie large ni en voie métrique mais en voie de $0,60 \mathrm{~m}$, à deux industriels locaux. La convention, passée entre le département et les concessionnaires le 4 mars 1893, est déclarée d'utilité publique le 25 juillet 1893. Dans le même temps, ils ont sollicité les communes concernées par les autres lignes du réseau départemental pour qu'elles votent des crédits correspondants à la moitié des frais d'études pour chaque kilomètre qui les traverseraient.

Toujours porté et défendu par Dubernard, le projet de réseau départemental sera satisfait, non sans quelques tentatives d'opposition d'élus républicains qui exigent qu'on respecte le principe de parité dans les dépenses d'études : le département en finance la moitié si les communes concernées en financent l'autre moitié.

Déstabilisés par ces exigences - certaines communes, peu peuplées et pauvres, qui seront traversées par de longues portions de la ligne de Castres à Murat, n'ont pas les moyens de payer la moitié des frais d'études -, Vergnes et Dubernard reçoivent le renfort d'Édouard Barbey :

«La règle posée me paraît [...] difficilement applicable d'une manière générale, tout au moins pour la région montagneuse de l'arrondissement de Castres. J'incline donc à penser que le département devrait faire les frais des études nécessaires. »

Mais les plus farouches élus républicains ne désarment pas : ils demandent maintenant que l'on procède à des études de rentabilité économique préalables et que l'on prenne des décisions pour l'ensemble du réseau et non pour telle ou telle ligne, au premier rang desquelles leurs attaques visent celle de la Montagne.

«Enterrement de $1^{\text {re }}$ classe », craint Dubernard. Une fois encore Barbey met un terme à la controverse qui se développe en faisant voter la charge des frais d'étude au département, en faisant élaborer par la Commission départementale la liste des huit lignes à étudier puis à construire.

L'affaire est bouclée le lendemain par un vote qui place en tête des priorités d'études et de construction la ligne de Castres à Murat. 
Cette décision intrigue... Dans un département maintenant largement dominé par les républicains (27 cantons contre 8 à la droite) (fig. 3), comment comprendre ce vote qui satisfait les intérêts de cinq cantons conservateurs (trois directement - Vabre, Brassac, Murat - et deux indirectement - Anglès et Montredon) et ceux de seulement deux cantons républicains (Roquecourbe et Lacaune) ? Comment comprendre que, après l'échec de René Reille au scrutin cantonal d'août 1889, son projet soit adopté avec le renfort de Barbey ? Dix ans après les affrontements entre Reille, Dubernard, Barbey et Compayré, l'alliance objective entre Dubernard et Barbey sur un sujet qui les a profondément divisés, a-t-elle un sens?

Remontons le temps.

Si le projet de réseau départemental est mis en sommeil pendant cinq ans par le conseil général, après le refus de Graulhet d'accepter la voie étroite, il n'est cependant pas oublié par les acteurs de ces premières joutes : les élections législatives de 1889 l'illustrent.

Édouard Barbey n'a jamais totalement rejeté le projet de desserte ferroviaire de la Montagne ; mais nous avons pu constater qu'il plaçait Graulhet en tête des priorités. Intérêts industriels et politiques se croisent : soutenir Graulhet contre la Montagne, soutenir, en 1883, le conservateur Abrial c'était œuvrer au développement industriel de Mazamet et contrer l'emprise politique de Reille...

En 1889, pour défier Reille, Barbey fait venir de Paris un candidat inattendu.

«Est-il neveu des Pereire comme on le dit ? Est-il juif - ce serait un comble !-comme on le prétend ? » : la plume du Conservateur se fait perfide...

Parent des Pereire, « ami de M. Carnot, président de la République », ingénieur centralien, maire du XVII ${ }^{e}$ arrondissement de Paris, membre du Comité consultatif des chemins de fer au ministère des Travaux publics, directeur de la Société générale des chemins de fer économiques, rétorque Le Réveil du Tarn pour présenter et défendre Émile Level qui vient défier René Reille. Le journaliste s'exclame :

«M. Level [...] n'est-il pas, plus que tout autre homme, en France, placé pour rendre à notre circonscription, des services généraux cent fois plus considérables? N'est-il pas le créateur, l'inventeur des chemins de fer économiques ? N'est-il pas le directeur de la Société générale des chemins de fer économiques? 》 (Réveil, 14 septembre 1889.) 


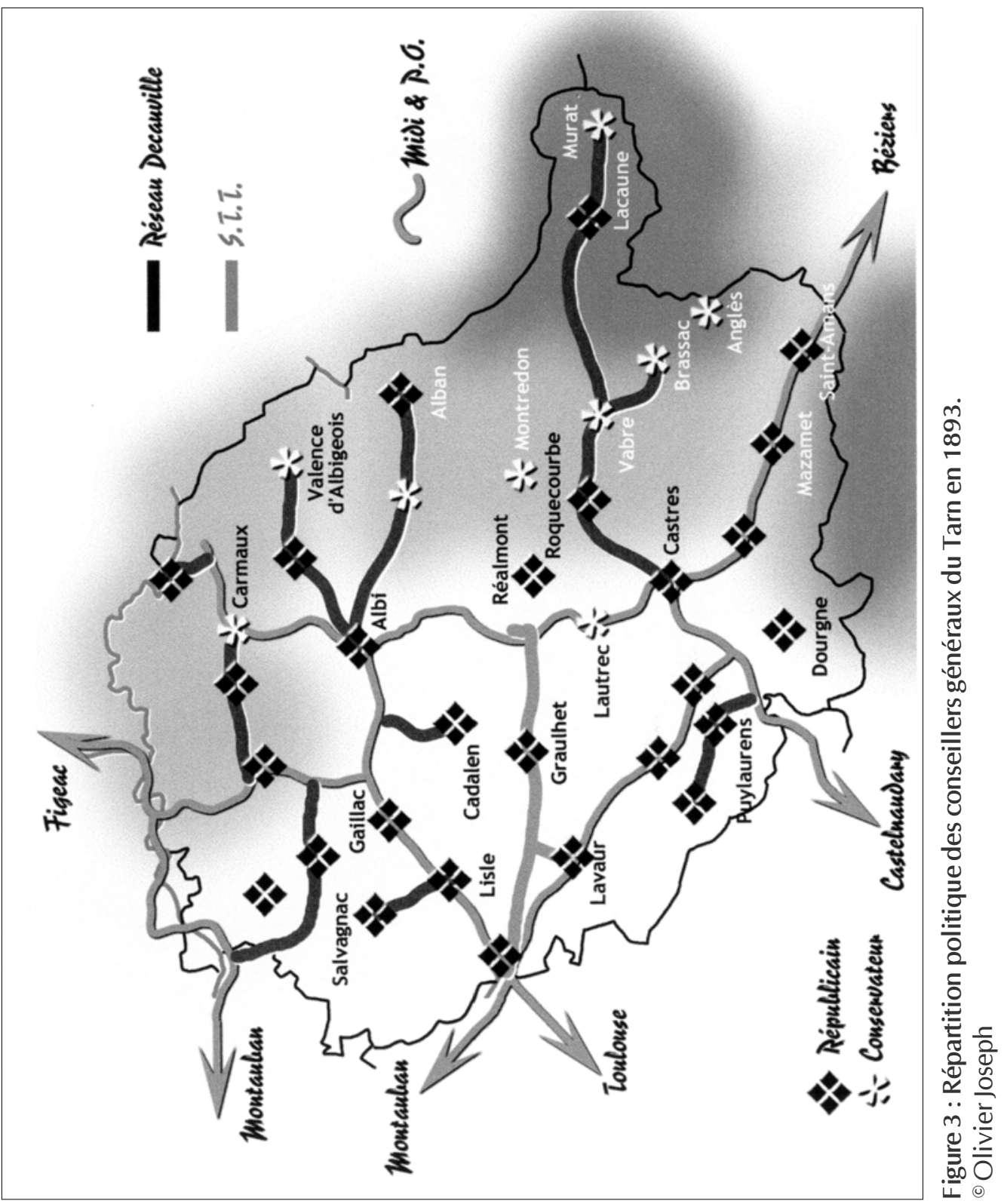


Les 5, 7, 8 et 9 septembre, Level et Barbey sont à Espérausse et Viane (canton de Lacaune), Murat et Moulin-Mage, à Vabre enfin. Partout, celui qui vient "pour tomber le roi de la Montagne» (Réveil, 11 septembre 1889) trouve un auditoire nombreux et attentif. Partout Level défend en premier lieu les lois de séparation de l'État et de l'Église. Partout aussi, Émile Level n’hésite pas à dire combien il est nécessaire de construire une voie ferrée pour pénétrer ces cantons et $\mathrm{y}$ « apporter la vie et le progrès » (Réveil, 14 septembre 1889). Emporté par l'ivresse électorale, il n'hésite pas à livrer ses conclusions d'expert :

"J'ai été même frappé en parcourant vos belles montagnes de voir avec quelle facilité on pourrait établir un chemin de fer [...] de Castres à Lacaune, en suivant les vallées, et combien il serait peu coûteux. » (Réveil, 14 septembre 1889.)

Foi de candidat, c'est l'ingénieur qui parle... Mais, prudent, le candidat-ingénieur ne manque d'ajouter:

«Je n’enverrai pas sur les lieux, avant les élections, une armée d’ingénieurs, pour planter des jalons et faire des études, car je déteste tout ce qui pourrait être taxé de manœuvre électorale ; mais ces études, je les ferai faire et de la façon la plus sérieuse, après les élections [...] (Réveil, 14 septembre 1889.)

Si le journal républicain a cru sentir le réveil des «populations agricoles de nos montagnes, qui commencent à comprendre que les vieilles routines ont fait leur temps » (14 septembre 1889), les urnes rendent un verdict contraire : René Reille est réélu député par 9934 voix contre 7463 à Émile Level.

Projet défendu par la droite du conseil général, la ligne de Castres à Murat entre, en 1889, dans le programme électoral des républicains, au même titre que la question de la séparation de l'État et de l'Église.

Mais c'est peut-être dans la circonscription adjacente de Castres que le sort définitif de la ligne de la Montagne va commencer, en grande partie, à se jouer. Élu sur un scrutin de liste en 1885, le candidat républicain Jean Jaurès affronte le fils Abrial. Le Conservateur ne saurait manquer l'occasion d'affûter sa plume :

« [...] il fait campagne non en simple pékin comme font généralement ses collègues, mais en grande tenue, en habit revêtu de ses insignes de député et porteur d'un couvre-chef [...] On dirait un arracheur de dents, un de ces dentistes ambulants couvert d'oripeaux et de ferreblanterie [sic] de toute sorte, qui courent nos foires et appellent l'attention du paysan à coup de grosse caisse et de tam-tam pour les exploiter et les duper.» (18 août 1889.) 
Le retour au scrutin uninominal fera certainement plus que les sarcasmes du Conservateur pour rendre à Abrial son siège de député et renvoyer Jean Jaurès à ses études... De 1889 à 1892, Jean Jaurès affirme ce qui, déjà, commençait à poindre et à effrayer certains notables castrais : son évolution vers le socialisme.

Voisins de circonscriptions en 1889, Reille et Jaurès vont croiser inextricablement leurs destins politiques durant l'année 1892.

Déplaçons-nous au nord-ouest du département, à Carmaux.

Depuis 1884, au titre de ses fonctions d'administrateur du ParisOrléans, René Reille préside les Mines de Carmaux. En 1888, les liens d'affaires entre les Solages - créateurs de la société des mines - et Reille se doublent de liens familiaux : Ludovic de Solages épouse Geneviève Reille, fille de René. Les deux familles consolident ainsi une alliance qui s'exprime dans plusieurs domaines : noblesse, capitalisme, cléricalisme, politique enfin, avec l'élection, en 1889, de Ludovic de Solages à l'Assemblée nationale.

1892... Élu maire de Carmaux à la tête d'une liste socialiste, Jean-Baptiste Calvignac est mis à pied le 2 août par la direction des Mines de Carmaux. Devant le refus de Solages et de Reille d'accorder à Calvignac la possibilité de remplir les obligations de son mandat pendant son temps de travail, les mineurs déclenchent, le 16 août, une grève destinée à durer dix semaines et à marquer l'histoire syndicale et politique française. L'intransigeance de Reille et de Solages, l'intervention de Jaurès portent le conflit à la Chambre où les interpellations socialistes forcent Reille à céder le 30 octobre. Ébranlé par les attaques dirigées contre lui, Solages démissionne de son mandat de député le 14 octobre, rouvrant à Jean Jaurès les portes de l'Assemblée nationale au terme d'une élection partielle en janvier 1893.

Lorsque Le Réveil du Tarn annonce, le 6 août, la candidature de Jean-Baptiste Calvignac contre René Reille dans la circonscription de Castres-Mazamet, on devine que l'affrontement entre les deux hommes se déplace du nord au sud du département, ce que confirment les premières réunions électorales du candidat socialiste : «Lutte personnelle de Calvignac contre le baron Reille, telle est l'impression qui se dégage des discours du candidat socialiste » note, discrètement, le très républicain Réveil (13 août 1993).

L'irruption d'un socialiste dans la géographie électorale si singulière de la circonscription de Castres-Mazamet, fait-elle vaciller Reille?

« [...] nulle part ailleurs les capitaux ne sont plus engagés qu'à Mazamet dans l'intérêt de la population ouvrière et [...] c'est à la 
suite des premières tentatives socialistes que Mazamet a vu ses manufactures décroitre. »

défend l'éditorialiste du Réveil du Tarn. L'adversaire serait donc le socialiste et non plus l'homme de « toutes les réactions »? Mais ce qui intrigue à la lecture du journal d'Édouard Barbey, c'est qu'on ne semble pas s'inquiéter outre mesure d'une absence de poids : entre Reille et Calvignac, il n'y a aucun candidat républicain !

Indétrônable, le « roi de la Montagne » obtient, le 20 août, un score proche de son niveau habituel : 9398 voix. Calvignac reste très en deçà des scores républicains avec 3136 voix. Mais le fait marquant du scrutin réside dans l'addition des abstentions, des bulletins blancs et nuls : 8496 voix... là où d'ordinaire on en compte environ 3500 !

La molle réaction des républicains face à l'absence de candidat de leurs rangs, l'abstention massive de leurs électeurs ont-elles un sens ?

Revenons quelques mois en arrière.

Catholique et conservateur, opposé à la République, le « roi de la Montagne », «l'homme de toutes les réactions », a été attentif au toast porté à Alger, en novembre 1890, par le cardinal Lavigerie. Devant les officiers de l'escadre de la Méditerranée, et à la demande du pape Léon XIII, la prélat invite ces catholiques monarchistes à accepter la République au nom de "l'amour de la patrie ». Face aux réticences de l'épiscopat français, Léon XIII publie, le 16 février 1892, sa seule encyclique rédigée en français, $A u$ milieu des sollicitudes. Le signal envoyé aux catholiques français est clair : la République est légitime et les catholiques sont « tenus » de l'accepter.

Dorénavant, plus par tactique que par réelle conviction, René Reille se range - non sans quelques ambiguités, comme le décrit Jean Faury dans sa thèse - du côté de la République et fait chanter $L a$ Marseillaise lors de ses réunions électorales !

Reste qu'ailleurs, on entonne avec aplomb et vigueur L'Internationale et La Carmagnole...

La coïncidence de la prise de position de Léon XIII et de la victoire des socialistes à Carmaux modifie le paysage politique tarnais. Républicains modérés et conservateurs «Ralliés » se trouvent engagés contre un même adversaire : les socialistes. Aux querelles politiques et religieuses se substitue une préoccupation commune dans la défense d'intérêts sociaux et économiques.

En août 1893, à Carmaux, Ludovic de Solages soutient le candidat républicain, Groc, contre Jaurès. À Mazamet, René Reille et Édouard Barbey concluent un accord tacite : au premier le siège de député, au 
second celui de sénateur. Les électeurs républicains mettent en œuvre l'accord en s'abstenant massivement, barrant ainsi la route à Calvignac.

Le 7 septembre 1893, le conseil général adopte définitivement le projet d'un réseau départemental secondaire de neuf lignes, au premier rang desquelles figure celle de Castres à Murat et Brassac, portée depuis plus de dix ans par les élus de droite.

Le 14 septembre, court-circuitant les procédures administratives, Édouard Barbey charge l'ingénieur en chef Georges Callon des études de ces lignes et le presse d'aboutir à des résultats conformes aux attentes de l'assemblée départementale.

Si l'année 1893 est, pour Jean Jaurès, une « sorte d'aurore » du socialisme en France, son élection au siège de Carmaux, la défaite de Jean-Baptiste Calvignac à Mazamet, le rapprochement de Reille et Barbey sont pareillement « une sorte d'aurore » pour la ligne qui, presque vingt ans plus tard, reliera effectivement Castres à la Montagne.

La décennie qui court d'avril 1883 à août 1893 a marqué l'histoire politique et religieuse du Tarn. Entre République naissante et volonté de restauration monarchique, entre État et Église, entre cléricaux et anticléricaux, les acteurs tarnais ont joué, à leur façon, une partition de l'histoire locale autant que nationale.

Le Petit Train qui semble, sous la plume des érudits locaux, naitre sereinement dans le ciel étoilé et pur des Idées ou du Progrès, est en fait porté par un vaste projet politique. Il se situe, durant ces dix années de discussions, à la charnière même des débats, des polémiques, des batailles qui agitent le Tarn et la France d'alors. Son existence faiblit ou forcit dans l'exacte mesure ou faiblissent ou forcissent les positions politiques de ses promoteurs. Son avenir se décide lorsque se recompose le paysage politique tarnais tout autant que français. En cela, il est indissociable de l'histoire de France et des destins croisés de ses plus ardents défenseurs comme de ses plus acharnés détracteurs.

Le temps des politiques semble terminé. D’autres acteurs vont venir au premier plan : les ingénieurs des Ponts et Chaussées, Georges Callon et son « cher camarade » du P.O. Paul Delzenne, Eugène Fouquet, Albert Hémardinquer, Rosario de Volontat, puis les concessionnaires, les frères Laborie, la famille Lemonnier et la Compagnie centrale de chemins de fer et de tramways... En leur compagnie, il faudra rendre visite à Paul Decauville, se pencher sur les courbes, contre-courbes, rebroussements et boucles du Darjeeling Himalaya Railway, sur les dunes du Sahara, sur le futur viaduc ferroviaire lyonnais entre Fourvières et Croix-Rousse, pénétrer les arcanes de la finance belge, user des fonds de culottes sur 
les bancs de Centrale et chez Thomson-Houston, devenir attentif à la naissance des chemins de fer des Côtes-du-Nord, à ceux du Beaujolais, aux Tramways d'Iekaterinoslaw dans la lointaine Russie, se pencher sur l'exploitation de ceux de Châteaubriant à Erbray... Tout cela entre 1893 et 1897, pour se retrouver, enfin, à la table des négociations préalables à l'attribution de la concession.

Me voici pris au piège des «destins croisés » d'Italo Calvino : il me faudra parcourir tous ces kilomètres, suivre tous ces lièvres qui m'entrainent bien loin des Monts de Lacaune et de ce charmant tortillard. Mais il me faudra le faire parce que les acteurs de ce qui ressemble de plus en plus à une épopée vont le faire pour, au bout de leurs peines, donner naissance à la ligne qui, soixante-dix ans durant, desservira le Haut-Languedoc tarnais.

Le Petit Train, dont la nostalgie est si forte dans les Monts de Lacaune, n'a ni ballast, ni traverse, ni rail, ni tire-fond, il ne bringuebale pas encore pour rejoindre Murat ou Brassac ; il lui faudra attendre encore plus de dix ans pour commencer à rouler. Déjà, pourtant, la France entière, le monde entier, semblent se pencher sur son berceau...

En va-t-il autrement ailleurs?

\section{Bibliographie}

Les documents de base de cette étude sont les Délibérations $d u$ conseil général du Tarn, lues systématiquement pour les années 1871 à 1901, ainsi que les collections des journaux Le Conservateur et Le Réveil du Tarn.

Sur la controverse scolaire et les oppositions entre cléricaux et anticléricaux, l'ouvrage indispensable demeure la thèse de Jean Faury, Cléricalisme et anticléricalisme dans le Tarn (1848-1900), Toulouse, Association des publications de l'université de Toulouse le Mirail, 1980.

Rémy Cazals, Avec les ouvriers de Mazamet dans la grève et l'action quotidienne, 1909-1914, Paris, Maspéro, 1978.

Rémy Cazals (sous la dir. de), Histoire de Castres, Maramet, la Montagne, Toulouse, Privat, 1992.

Rolande Trempé, Les Mineurs de Carmaux, 1848-1914, Paris, Éditions Ouvrières, 1971.

Rolande Trempé, « Du Royalisme à la République ou le "ralliement" du Marquis de Solage, député de la $2^{\mathrm{e}}$ circonscription d'Albi (Tarn) ", Annales du Midi, 1959, pp. 59-70. 
Maurice Greslé-Bouignol (sous la dir. de), Les Tarnais. Dictionnaire biographique, Fédération des Sociétés Intellectuelles du Tarn, 1996.

Guy Viala (sous la dir. de), Il était une fois le Petit Train, Société Culturelle du Pays Castrais, 1987.

Pierre Gaches, Le Petit Train de Castres à Murat et à Brassac, Toulouse, 1972.

François Caron, La France des patriotes, Paris, Fayard, 1985 (t. V de l'Histoire de France dirigée par Jean Favier)..

Harvey Goldberg, Jean Jaurès, Paris, Fayard, 1970.

Jean-François Sirinelli (sous la dir. de), Histoire des droites en France, Paris, Gallimard, 1992.

Gérard Cholvy et Yves-Marie Hilaire, Histoire religieuse de la France, Toulouse, Privat, 1986.

\section{Notes}

1. Thomas Hughes, Networks of Power. Electrification in Western Society, 18801930, Baltimore, John Hopkins University Press, 1983.

Donald Mackenzie, Inventing Accuracy. A historical sociology of nuclear missile guidance, Cambridge, MIT Press, 1990.

Bruno Latour, Aramis ou l'amour des techniques, Paris, La Découverte, 1992. La Science en action, Paris, La Découverte, 1989.

On pourra lire aussi :

Wiebe Bijker, Thomas Hughes, Trevor Pinch (sous la dir. de), The Social Construction of Technological Systems, Cambridge, MIT Press, 1987.

Wiebe Bijker, John Law, Shaping Technology/Building Society, Cambridge, MIT Press, 1992.

Enfin, bien qu'elles concernent les sciences et non les techniques, l'étude de Martin Rudwick ou celle de Steven Shapin et Simon Schaffer sont riches d'enseignements quant à l'intérêt qu'il y a à suivre les méandres d'une controverse scientifique : The Great Devonian Controversy. The shaping of scientific knowledge among gentlemanly specialists, Chicago University Press, 1985 ; Leviathan and the Air-Pump: Hobbes, Boyle and the Experimental Life, Princeton University Press, 1985.

2. Et je limite ce temps des politiques à la seule construction « politicienne » de la décision. Une lecture attentive des sources disponibles permet d'esquisser les mondes que portent en eux les défenseurs de telle ou telle solution : entre déplacements, développement économique, maitrise et organisation du territoire et de ses populations, chaque camp est porteur d'un véritable projet social et politique. 